\title{
Clinical and biochemical responses after Gamma Knife surgery for a dopamine-secreting paraganglioma: case report
}

\author{
Constantin Tuleasca, ${ }^{1,2,9}$ Yves Jaquet, ${ }^{3}$ Valerie Schweizer, ${ }^{3}$ Laura Negretti, ${ }^{4}$ \\ Vera Magaddino, ${ }^{5,9}$ Philippe Maeder, ${ }^{6,9}$ Karim-Alexandre Abid, ${ }^{8,9}$ Benoit Lhermitte, ${ }^{7,9}$ \\ Eric Grouzmann, ${ }^{* 8,9}$ Marc Levivier*1,9
}

\begin{abstract}
${ }^{1}$ Department of Clinical Neurosciences, Neurosurgery Service and Gamma Knife Center, Centre Hospitalier Universitaire Vaudois (CHUV); ${ }^{2}$ Signal Processing Laboratory (LTS 5), École Polytechnique Fédérale de Lausanne; ${ }^{3}$ Department of Otolaryngology, Head and Neck Surgery, ${ }^{4}$ Radiation Oncology Service, ${ }^{5}$ Institute of Radiation Physics, ${ }^{6}$ Radiology Department, ${ }^{7}$ Neuropathology Department, ${ }^{8}$ Service of Biomedicine, Laboratoire des Catécholamines et Peptides, Centre Hospitalier Universitaire Vaudois (CHUV); ${ }^{9}$ University of Lausanne, Faculty of Biology and Medicine; Lausanne, Switzerland
\end{abstract}

*These authors contributed equally to this work

\begin{abstract}
INTRODUCTION: The efficacy of Gamma Knife surgery (GKS) in local tumor control of non-secreting paragangliomas (PGLs) has been fully described by previous studies. However, with regard to secreting PGL, only one previous case report exists advocating its efficacy at a biological level. CASE REPORT: The aims of this study were: 1) to evaluate the safety/efficacy of GKS in a dopamine-secreting PGL; 2) to investigate whether the biological concentrations of free methoxytyramine could be used as a marker of treatment efficacy during the follow-up. We describe the case of a 62-year-old man diagnosed with left PGL. He initially underwent complete surgical excision. Thirty months after, he developed recurrent biological and neuroradiological disease; the most sensitive biomarker for monitoring the disease, concentration of plasma free methoxytyramine, started to increase. GKS was performed at a maximal marginal dose of $16 \mathrm{~Gy}$. During the following 30 months, concentration of free methoxytyramine gradually decreased from $0.14 \mathrm{nmol} / \mathrm{l}(2 * \mathrm{URL})$ before GKS to $0.09 \mathrm{nmol} / \mathrm{l}, 6 \mathrm{months}$ after GKS and $0.07 \mathrm{nmol} / \mathrm{l}$ at the last follow-up after GKS $(1.1 * \mathrm{URL})$, confirming the efficacy of the treatment. Additionally, at 30 months there was approximately $36.6 \%$ shrinkage from the initial target volume. CONCLUSION: The GKS treatment was safe and effective, this being confirmed clinically, neuroradiologically and biologically. The case illustrates the importance of laboratory tests taking into account methoxytyramine when analyzing biological samples to assess the biochemical activity of a PGL. In addition, the identification of methoxytyramine as a unique positive biomarker could designate it for the monitoring of tumor relapse after treatments, including Gamma Knife surgery.
\end{abstract}

Key words: Dopamine, Gamma Knife surgery, Paraganglioma, Secreting

Address for correspondence:

Constantin Tuleasca, MD; MD-PhD candidate, Lausanne University Hospital, Department of Clinical Neurosciences, Neurosurgery Service and Gamma Knife Center, Rue de Bugnon 44-46, BH-08, CH-1011, Lausanne, Switzerland; Tel.: +41-21-314-26-02,

Fax: +41-21-314-11-99, E-mail: constantin.tuleasca@gmail.com

Received: 29-04-2015, Accepted: 06-07-2015 


\section{INTRODUCTION}

Paragangliomas (PGLs) are neuroendocrine tumors, slow growing and arising principally in the head and neck. In $1-2 \%$ of cases they comprise functional, secreting catecholamines entities. ${ }^{1}$ Considered generally benign, they however include 1-5\% malignancies. ${ }^{2}$ Due to their topography and local invasion of surrounding structures, they are associated with a complexity of clinical signs and symptoms (headache, pulsatile tinnitus, lower cranial nerve palsies). Therefore, their treatment remains challenging and includes a variety of alternatives, including surgical resection, embolization and radiotherapy. Stereotactic radiosurgery has recently emerged as a non-invasive alternative for the treatment of head and neck PGL, demonstrating high rates of local tumor control and symptoms with by minimal associated morbidity. ${ }^{3-7}$

Functional PGLs secreting catecholamines are rather rare entities. Hypertension is present in 95\% of them. ${ }^{8}$ While the sensitivities reported in patients with catecholamine-secreting PGLs are 74\% for urine total metanephrines, $84 \%$ for norepinephrine, $18 \%$ for dopamine and $14 \%$ for epinephrine, unfortunately no sensitivity has thus far been reported for methoxytyramine ${ }^{8}$ However, it appears that free methoxytyramine is virtually absent in plasma and any presence is associated with an intratumoral metabolism of dopamine into its O-methoxylated form, methoxytyramine by catechol-O-methyltransferase (COMT) ${ }^{8}$

In the rare setting of functional PGLs, only one case report of radiosurgical treatment has to date been published. ${ }^{3}$

We present the case of an adult male with a recurrent dopamine-secreting PGL, biochemically monitored by measuring urine and plasma free methoxytyramine, that was treated successfully with GKS for a postsurgical recurrence. The tumor was not producing normetanephrine nor metanephrine. ${ }^{9}$

\section{CASE REPORT}

\section{Initial presentation and work-up}

A 62-year-old man was referred to an otolaryngologist in July 2004 for investigation of a left cervical mass. After a clinical examination, the otolaryngolo- gist considered the mass to be a lipoma and no biopsy was performed. No other investigation was performed for the next 4 years.

In April 2008, the patient complained of cervical pain and noted an increase of the cervical mass. His general practitioner ordered a computed tomography scan of the neck that revealed a hypervascular $3.5 \mathrm{x}$ $3.8 \mathrm{~cm}$ mass in the left carotid region. A fine-needle aspiration biopsy was not conclusive and showed only hemorrhagic material.

In May 2008, a magnetic resonance (MR) imaging scan with MR angiography sequences was carried out, which showed a $4 \times 4 \times 4.7 \mathrm{~cm}$ left latero-cervical strongly contrast-enhancing mass posterior to the carotid artery. His medical history was significant for an arterial hypertension diagnosed in June 2002 and treated by an angiotensin II receptor blocker (ARB) and a thiazide diuretic, but no specific symptoms suggestive of catecholamine excess were reported. The patient was scheduled for surgical removal of the mass.

A ${ }^{123}$ I-Meta-iodobenzylguanidine ( ${ }^{123}$ I-MIBG) scintigraphy did not reveal uptake through norepinephrine transporters, a feature previously reported for dopamine-secreting tumors, probably due to the absence of the vesicular monoamine transporter VMAT. ${ }^{1}$ ${ }^{18} \mathrm{~F}$-fluoro-D-glucose Positron Emission Tomography (FDG-PET) confirmed the localization of the known mass, which had an increased FDG uptake but did not invade other tissues. PET also revealed a centrimetric left jugular lymph node and multiple bone lesions in the thoracic and lumbar spine, all without increased uptake. Findings on a subsequent bone scintigraphy and a total-body MR imaging were not compatible with metastatic lesions.

In August 2008, the patient underwent a gross total resection of the mass. Blood pressure and heart rate were unremarkable throughout the surgical procedure, confirming that the tumor was not secreting vasoactive bioamines and peptides since no changes of blood pressure or heart rate were observed as is the case in the surgical removal of pheochromocytomas. The arterial hypertension still persisted after tumor removal and was adequately controlled by an ARN and a thiazide diuretic, this being indicative of essential hypertension. The histopathological ex- 
amination of the tumor confirmed the diagnosis of PGL. The tumor margins reached the resection area on many sites and tumoral cells presented a marked pleomorphism with few mitoses (one mitosis per 10 fields at high magnitude).

The possibility of a biochemically functional tumor was evaluated by 24-hour urine and plasma measurements for metanephrines and catecholamines. A urine specimen collection was also performed.

\section{Surgical intervention and initial surveillance}

The urine collection showed elevated concentrations of nmol/24h methoxytyramine at 10880 (Upper Reference Limit, URL <1900) and a relatively high concentration of dopamine but still within the normal range at 3222 (URL <3300). Similarly, plasma free and total methoxytyramine and dopamine were increased to 2.78, 26.90 and $20.46 \mathrm{nmol} / \mathrm{L}$ (URL $<0.06,2.99$ and 0.38 , respectively). Norepinephrine and epinephrine and their methoxylated metabolites were within their respective reference intervals in urine and plasma. ${ }^{9}$ Routine blood analysis was unremarkable. A new $24 \mathrm{~h}$ urinary sample confirmed increased levels of methoxytyramine at 12704 and dopamine at $3547 \mathrm{nmol} / 24 \mathrm{~h}$. Genetic testing for mutations of the succinate dehydrogenase genes SDHD and SDHB was negative.

A piece of tumor tissue was used for biochemical analysis and demonstrated an abundant production of dopamine and methoxytyramine ( 25.3 and $6.4 \mathrm{pmol} / \mathrm{mg}$ of wet tissue, respectively), along with low amounts of norepinephrine and epinephrine $(0.87$ and $0.87 \mathrm{pmol} /$ $\mathrm{mg}$ ) and of normetanephrine and metanephrine (0.04 and $0.4 \mathrm{pmol} / \mathrm{mg}$ ), indicative of decreased expression of dopamine beta-hydroxylase, the enzyme that transforms dopamine into norepinephrine.

The patient recovered and 5 days post-surgery biochemical evaluation showed catecholamines and metabolites within normal ranges, as they remained during the last 2.5 years of the patient's annual followup. A left Claude-Bernard-Horner syndrome, with additional partial palsy of the left 11th and 12th nerves, had marked the clinical postsurgical course.

The biological sample analyzed in July 2010 (2 years after surgery) showed a concentration of plasma free methoxytyramine below the URL at $0.05 \mathrm{nmol} / 1$
$(\mathrm{URL}<0.06)$ and low urine methoxytyramine at 1211 nmol/24 hr (URL at 1900). In February 2011 (2 years and a half after surgery), urinary and plasmatic levels of methoxytyramine were measured and started to increase (1393 and 0.10, respectively). A new FDGPET was compatible with a recurrence of the PGL in the region of the left carotid and also revealed a lesion in the vocal chords with increased uptake. A MR confirmed a $1.7 \times 1 \times 1.2 \mathrm{~cm}$ lesion, situated in the left retrosytloid parapharyngeal space, which was hyperintense on T2, but no other lesions. Therefore, a neuroradiological and biochemical recurrence was diagnosed.

The anatomical location of the recurrent tumor, and particularly its close proximity to the internal carotid artery, represented a high surgical risk in the event of a new open microsurgical intervention. After multidisciplinary discussion regarding surgical or radiation management, a GKS treatment was decided upon.

\section{Radiosurgical salvage therapy and postoperative course}

Gamma Knife surgery was performed in December 2011, after placement of the stereotactic Leksell Frame type G, with the Leksell Gamma Knife Perfexion ${ }^{\circledR}$ (Elekta Instruments, AB, Sweden) (Figure 1a). The lesion size was $20 \mathrm{~mm}$ (lateral), $19 \mathrm{~mm}$ (antero-posterior) and $42 \mathrm{~mm}$ (vertical) at the time of GKS. The target volume was $6.64 \mathrm{cc}$. The maximum marginal dose was $16 \mathrm{~Gy}$ at the $50 \%$ prescription isodose. The prescription isodose volume was $9.79 \mathrm{cc}$. The conformity, the selectivity, the Paddick and the gradient index were 1, 1.694, 1.694 and 3.122, respectively. Eighteen isocenters were used (including composite ones). The dose gradient was optimized towards the interface with the ipsilateral internal carotid artery and also towards the jugular foramen, as the tumor was infiltrating the former.

The follow-up course included regular clinical, biochemical and neuroradiological assessment. The latest available information after GKS was at 30 months.

Seven months after GKS, no new symptom was present. There was persistent partial palsy of the left 11 th and 12 th nerves. Biochemically, the urinary and 


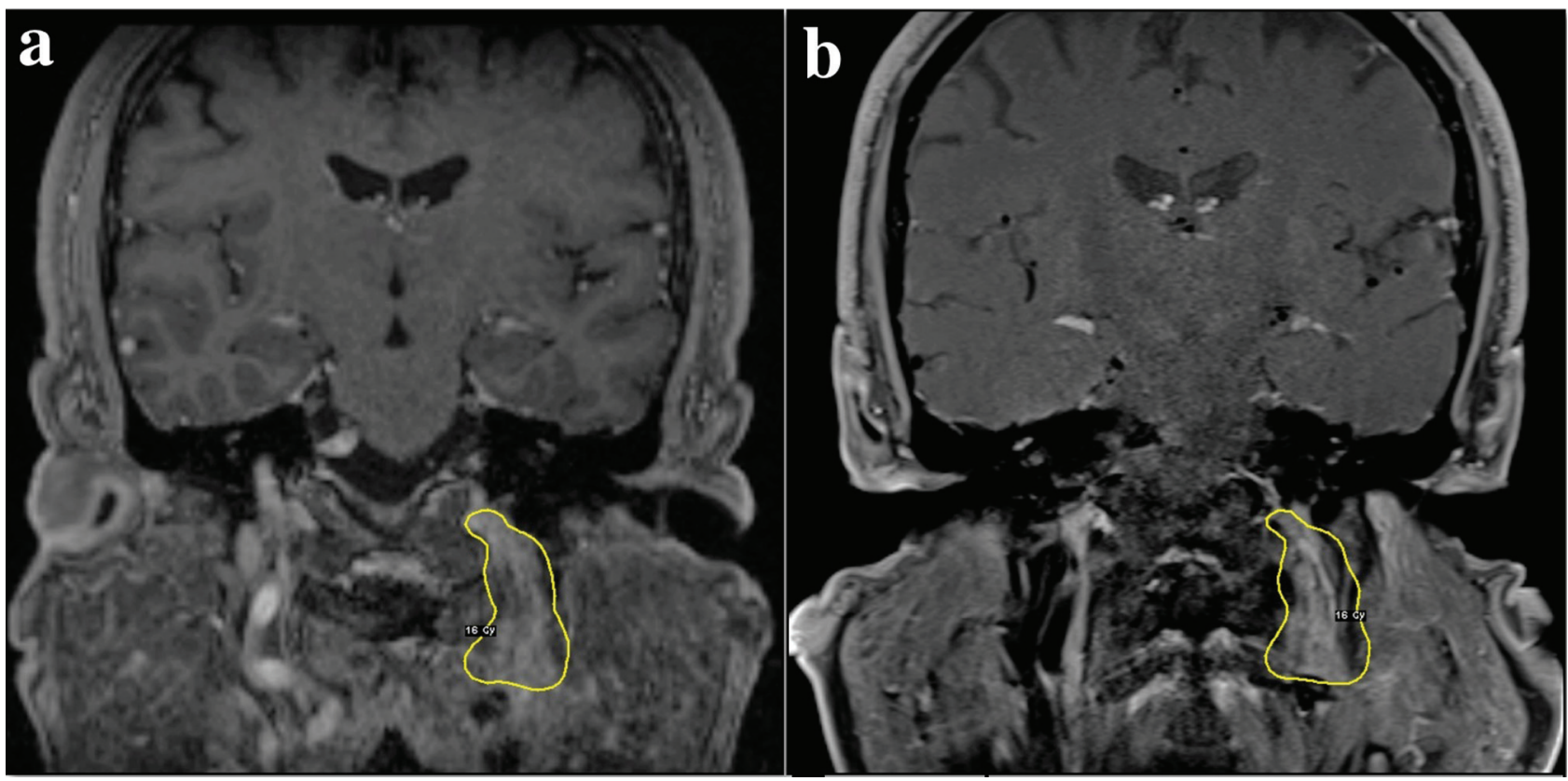

Figure 1. T1 weighted MR imaging with Gadolinium in the coronal plane, at the moment of GKS (a) and 30 months after (b), showing shrinkage of the PGL. The tumor is enclosed by the 16 Gy (50\%) isodose line.

plasmatic levels of catecholamines were discretely diminishing. Neuroradiologically, there was stability of the tumor size and aspect.

The patient underwent surveillance imaging, serology and clinical assessment at 19 and 30 months after salvage radiosurgery and remained clinically stable and without new neurologic deficits. Biochemically, the plasmatic concentration of free methoxytyramine gradually declined from $0.14 \mathrm{nmol} / 1$ ( $2 * \mathrm{URL})$ before GKS to $0.07 \mathrm{nmol} / 130$ months after GKS (1.1*URL), confirming the efficacy of the treatment (Table 1 and
Figure 2). Furthermore, their urine concentration from 1803 (before GKS) was decreased to $1232 \mathrm{nmol} / 1$ (at 30 months after GKS), a 31.7\% decline compared to pre-surgical values (Figure 2). The MR already showed shrinkage of the tumor at 19 months, which persisted over time (Figure 1b). At the last MRI, the remnant tumor volume measured $4.21 \mathrm{cc}$, which corresponded to a shrinkage of approximately $36.6 \%$ from the initial target volume (as calculated in the Leksell GammaPlan station, Elekta Instruments, AB, Sweden, the same as for the measurement of the initial GKS target volume).

Table 1. Measured plasma free and urine methoxytyramine concentrations during monitoring of the disease, urinary levels, nmol/24h

\begin{tabular}{lcr}
\hline Date & Plasma free methoxytyramine & Urine methoxytyramine \\
\hline July 2008 (Before surgery) & 2.78 & 12704 \\
September 2008 (Immediate post surgery) & $<0.03$ & 617 \\
July 2010 (16 months before GKS ) & 0.05 & 1211 \\
February 2011 (10 months before GKS) & 0.10 & 1393 \\
March 2011 (9 months before GKS) & 0.14 & 1803 \\
June 2012 (6 months post-GKS) & 0.09 & 1271 \\
June 2013 (18 months post-GKS) & 0.06 & 1329 \\
June 2014 (30 months post-GKS) & 0.07 & 1232 \\
\hline
\end{tabular}


Plasma free methoxytyramine

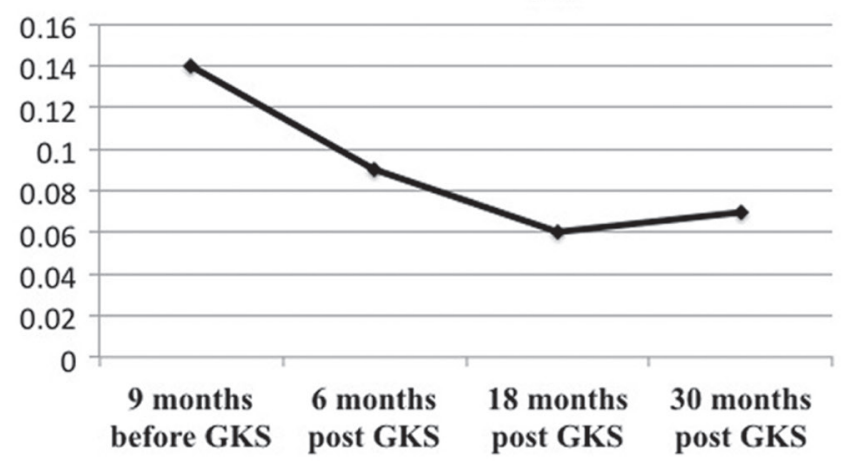

Urine methoxytyramine

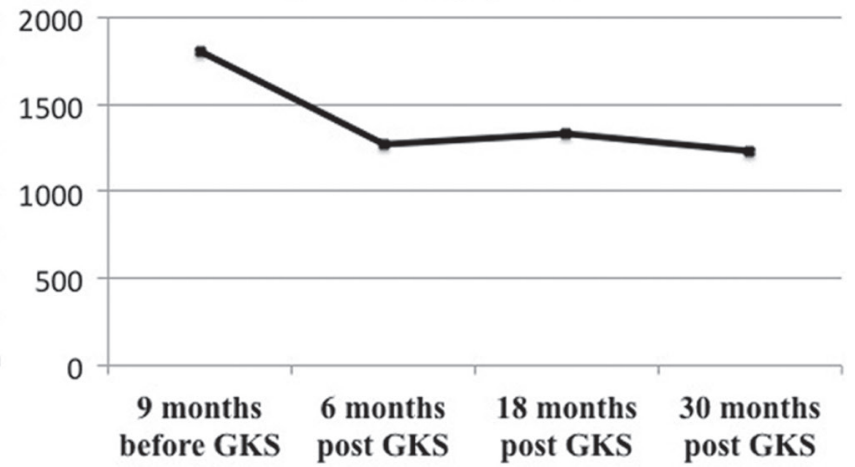

Figure 2. Plasma concentration (left panel) of free methoxytyramine gradually declined from $0.14 \mathrm{nmol} / \mathrm{l}$ (2*URL) before GKS to 0.07 $\mathrm{nmol} / 1$ and 30 months after GKS (1.1*URL); its urine concentration (right panel) decreased from 1803 (before GKS) to $1232 \mathrm{nmol} / 1$ (at 30 months after GKS), a 31.7\% change compared with the values before GKS.

\section{DISCUSSION}

Head and neck PGLs arise mostly sporadically, but they can also be familial (10-15\% of cases). ${ }^{10}$ They can be found in 3 main areas: most commonly around the bifurcation of the common carotid artery, less commonly within or along the inferior nodose ganglion of the 5th cranial nerve and, lastly, also along the branches of 4th and 5th nerve, in close association with the temporal bone, primarily in and around the jugular foramen (these latter known as glomus jugular tumors or chemodectoma). Glomus jugular tumors are extremely rare, with an incidence of about 1 in 30,000 in the healthy population, representing $0.0012 \%$ of all tumors diagnosed in humans. ${ }^{7}$ They are usually slow growing, with a mean growth rate of 0.8 to $1 \mathrm{~mm}$ per year. ${ }^{11}$ Glomus jugular tumors are highly vascular tumors; they grow expansively, eroding the jugular foramen and the petrous bone and they commonly gain access to the subarachnoid space by penetrating the dura of the posterior fossa or, less commonly, the middle fossa. ${ }^{12}$

The first surgical excision for this type of tumor was performed in 1945 by Rosenwasser. ${ }^{13}$ During the last few decades, progress in the surgical treatment of these tumors has been marked by some controversy due to significant morbidity associated with complete surgical excision, ${ }^{12,14}$ including up to $59 \%$ postoperative cranial nerve deficits. ${ }^{12}$ In subtotal resections, as reported by several studies, up to $15 \%$ recurrences are reported..$^{12,14,15}$
In this context, the need for minimally invasive therapies appeared. Embolization is not considered curative due to formation of anastomotic blood vessels and is recommended only as a preparation for surgery. ${ }^{16}$ Meanwhile, external-beam radiotherapy presents the risk of serious complications due to large fields size, as suggested by Li et al. ${ }^{17}$

Gamma Knife surgery emerged as an alternative during the last decade due to its minimal invasiveness and local tumor control rates comparable to surgical strategies, while preserving or even improving cranial nerve function..$^{4-6,18}$

Recently it has been reported that functioning PGLs also respond to stereotactic radiosurgery, and in particular to GKS, at both the biochemical and neuroradiological level. ${ }^{3}$ In addition, previously published papers have suggested that conventional doses and techniques of radiotherapy are not sufficient to adequately control these particular and rare cases. ${ }^{19-21}$

Functioning PGLs are very rare in the head and neck, although they are known to display neuroendocrine activity through secretion of catecholamines. ${ }^{10,22}$ The sensitivities reported in patients with catecholaminesecreting PGLs are $74 \%$ for urine total metanephrines, $84 \%$ for norepinephrine, $18 \%$ for dopamine and $14 \%$ for epinephrine, but unfortunately no sensitivity has as yet been reported for methoxytyramine. ${ }^{8} \mathrm{~A}$ recent study on a cohort of 136 patients with PGLs showed catecholamines secretion only in $23 \% .{ }^{23}$ The isolated secretion of methoxytyramine by PGLs is limited to 
$8-13 \%$ of these tumors. ${ }^{23,24}$ Thus, the tumors under discussion represent a real challenge for biochemical diagnosis, since medical laboratories usually do not report dopamine and its methoxylated derivative in plasma and urine samples. Therefore, chromatograms must be carefully inspected in order not to miss the diagnosis. In the present case, urinary dopamine appeared initially, at diagnosis, to be negative in one sample and slightly above the URL in a second sample, contrasting with the clear-cut increases above URL of plasma free (46-fold) and total methoxytyramine (9-fold), urine methoxytyramine (6-fold) and plasma dopamine (54-fold), in agreement with previous observations. ${ }^{23,24}$ Urinary dopamine deriving from renal extraction and decarboxylation of circulating 3,4-dihydroxyphenylalanine, found in certain kinds of foods and herbs, and by L-DOPA treatment is common in patients treated for Parkinson's disease, thus leading to false positive results. ${ }^{25}$ The higher diagnostic sensitivity of methoxytyramine for HNPGL is due to the fact that this tumor expresses catecholO-methyl-transferase, an enzyme that O-methylates intra-tumoral dopamine into methoxytyramine.

\section{CONCLUSION}

The present case illustrates the important need for laboratory tests to take into account methoxytyramine when analyzing biological samples to assess the biochemical activity of a PGL. In addition, the identification of methoxytyramine as being the only positive biomarker in such patients could designate it as a valuable biomarker for the monitoring of tumor relapse.

The GKS treatment was safe and effective, which was confirmed clinically, neuroradiologically and biochemically. To the best of our knowledge, this is the first report using methoxytyramine for the biological follow-up course of the treatment.

\section{SUPPORT}

Lausanne University Hospital.

\section{CONFLICT OF INTEREST}

The authors report no conflict of interest.

\section{REFERENCES}

1. Kuhweide R, Lanser MJ, Fisch U, 1996 Catecholaminesecreting paragangliomas at the skull base. Skull Base Surg 6: 35-45.

2. Manolidis S, Shohet JA, Jackson CG, Glasscock ME $3^{\text {rd }}, 1999$ Malignant glomus tumors. Laryngoscope 109: 30-34.

3. Castrucci WA, Chiang VL, Hulinsky I, Knisely JP, 2010 Biochemical and clinical responses after treatment of a catecholamine-secreting glomus jugulare tumor with gamma knife radiosurgery. Head Neck 32: 1720-1727.

4. Chen PG, Nguyen JH, Payne SC, Sheehan JP, Hashisaki GT, 2010 Treatment of glomus jugulare tumors with gamma knife radiosurgery. Laryngoscope 120: 18561862.

5. Lieberson RE, Adler JR, Soltys SG, Choi C, Gibbs IC, Chang SD, 2012 Stereotactic radiosurgery as the primary treatment for new and recurrent paragangliomas: is open surgical resection still the treatment of choice? World Neurosurg 77: 745-761.

6. Miller JP, Semaan M, Einstein D, Megerian CA, Maciunas RJ, 2009 Staged Gamma Knife radiosurgery after tailored surgical resection: a novel treatment paradigm for glomus jugulare tumors. Stereotact Funct Neurosurg 87: 31-36.

7. Schwaber MK, Glasscock ME, Nissen AJ, Jackson CG, Smith PG, 1984 Diagnosis and management of catecholamine secreting glomus tumors. Laryngoscope 94: 1008-1015.

8. Erickson D, Kudva YC, Ebersold MJ, et al, 2001 Benign paragangliomas: clinical presentation and treatment outcomes in 236 patients. J Clin Endocrinol Metab 86: 5210-5216.

9. Puder J, Stadelmann R, Schoettker P, Buclin T, Grouzmann E, 2013 A pain in the neck. Clin Chem 59: 12801281.

10. Woods CI, Strasnick B, Jackson CG, 1993 Surgery for glomus tumors: the Otology Group experience. Laryngoscope 103: 11 Pt 2 Suppl 60: 65-70.

11. Jansen JC, van den Berg R, Kuiper A, van der Mey AG, Zwinderman AH, Cornelisse CJ, 2000 Estimation of growth rate in patients with head and neck paragangliomas influences the treatment proposal. Cancer 88: 2811-2816.

12. Jackson CG, Kaylie DM, Coppit G, Gardner EK, 2004 Glomus jugulare tumors with intracranial extension. Neurosurg Focus 17: E7.

13. Rosenwasser H, 1952 Glomus jugularis tumor of the middle ear; carotid body tumor, tympanic body tumor, nonchromaffin paraganglioma. Trans Am Laryngol Rhinol Otol Soc 3(56th Meeting): 94-106.

14. Al-Mefty O, Teixeira A, 2002 Complex tumors of the glomus jugulare: criteria, treatment, and outcome. J Neurosurg 97: 1356-1366. 
15. Green JD Jr, Brackmann DE, Nguyen CD, Arriaga MA, Telischi FF, De la Cruz A, 1994 Surgical management of previously untreated glomus jugulare tumors. Laryngoscope. 104: 8 Pt 1: 917-921.

16. Tasar M, Yetiser S, 2004 Glomus tumors: therapeutic role of selective embolization. J Craniofac Surg 15: 497-505.

17. Li G, Chang S, Adler JR Jr, Lim M, 2007 Irradiation of glomus jugulare tumors: a historical perspective. Neurosurg Focus 23: E13.

18. Genc A, Bicer A, Abacioglu U, Peker S, Pamir MN, Kilic T, 2010 Gamma knife radiosurgery for the treatment of glomus jugulare tumors. J Neurooncol 97: 101-108.

19. Duke WM, Phillips MW, Donald JM Jr, Boshell BR, 1965 A Norepinephrine-Secreting Glomic Tissue Tumor (Chemodectoma). JAMA 193: 20-22.

20. Pluta RM, Ram Z, Patronas NJ, Keiser H, 1994 Longterm effects of radiation therapy for a catecholamine- producing glomus jugulare tumor. Case report. J Neurosurg 80: 1091-1094.

21. Osorio JE, Powell TD, Frank RS, et al, 2003 Recombinant raccoon pox vaccine protects mice against lethal plague. Vaccine 21: 1232-1238.

22. Brown JS, 1985 Glomus jugulare tumors revisited: a ten-year statistical follow-up of 231 cases. Laryngoscope 95: 284-288.

23. van Duinen N, Steenvoorden D, Kema IP, et al, 2010 Increased urinary excretion of 3-methoxytyramine in patients with head and neck paragangliomas. J Clin Endocrinol Metab 95: 209-214.

24. Eisenhofer G, Goldstein DS, Sullivan P, et al, 2005 Biochemical and clinical manifestations of dopamineproducing paragangliomas: utility of plasma methoxytyramine. J Clin Endocrinol Metab 90: 2068-2075.

25. Zendron L, Fehrenbach J, Taverna C, Krause M, 2004 Pitfalls in the diagnosis of phaeochromocytoma. BMJ 328: 629-630. 